\title{
COMBINED FOCUSED ION BEAM AND SECONDARY ION MASS SPECTROMETRY FOR HIGH RESOLUTION LIGHT ELEMENT DETECTION APPLIED ON LI-ION BATTERIES
}

Gudrun Wilhelm ${ }^{1}$, Ute Golla-Schindler ${ }^{2}$, Katharina Wöhrl ${ }^{3}$, Christian Geisbauer ${ }^{4}$, Graham Cooke ${ }^{5}$, Timo Bernthaler $^{6}$ and Gerhard Schneider ${ }^{6}$

${ }^{1}$ 1. Materials Research Institute (IMFAA), Aalen University, Aalen, Germany, Aalen, Baden-Wurttemberg, Germany, ${ }^{2} 1$. Materials Research Institute (IMFAA), Aalen University, Aalen, Germany, Aalen, Germany, ${ }^{3} 1$. Abteilung ZAF/CARISSMA, Technische Hochschule Ingolstadt, Ingolstadt, Germany, Ingolstadt, Bayern, Germany, ${ }^{4} 1$. Abteilung ZAF/Carissima, Technische Hochschule Ingolstadt, Ingolstadt, Germany, Aalen, Bayern, Germany, ${ }^{5}$ Hiden Analytical GmbH, Düsseldorf, Germany, Germany, ${ }^{6} 1$. Materials Research Institute (IMFAA), Aalen University, Aalen, Germany, United States

The combination of Secondary Ion Mass Spectrometry (SIMS) with Scanning Electron Microscopy (SEM) enables high-resolution spatially resolved surface analysis. A gallium primary ion beam removes neutral atoms, positive and negative ionised molecule fragments and element ions with their isotopic fingerprint. The detectable signal depends on the surface roughness, the material composition of the sample, the sputter rate and the instrument settings and performance. A polished graphite sample was selected as standard (comparable to anode foils in Li-ion batteries) to obtain the first insights on the dependence of the instrument settings. The main measurements were performed with a ZEISS Crossbeam 540 combined with a HIDEN Secondary Ion Mass Spectrometer (SIMS). Alternatively a combination with a ZEISS Time of Flight - Secondary Ion Mass Spectrometer (ToF-SIMS) was used. The Hiden SIMS allows serial measurements with high mass resolution while the ToF-SIMS enables a simultaneous measurement of all elements. The correlation between element intensity and gallium ion beam current is shown in Fig. (1). Beam current increase results in intensity increase of the peaks and therefore the total intensity Fig. 1c) and higher sample removal Fig. 1d). Large measuring fields (e.g. $28 \mu \mathrm{m}^{2}$, green curve Fig.1d)) show no significant sample removal increase and enable surface sensitive measurements.

SIMS is a powerful tool to complete analytical investigations on aged lithium ion batteries as this overcomes the bottleneck of lithium detection. At the interface between anode and electrolyte, the first two cycling processes induce the formation of the solid electrolyte interface (SEI) [1] which undergoes dynamic changes with battery ageing [2]. Two investigations were performed on commercial graphite/NMC (18650 and pouch cells). Ageing took place by storage at $60^{\circ} \mathrm{C}$ (73 days) and by cycling with $1 \mathrm{C}(1024$ cycles, $\mathrm{SOH} 88 \%)$. Storage ageing with electrochemical tests was carried out at the Technische Hochschule Ingolstadt. The anode surface of the storage cell shows strong incrustation (Fig 2a-b marked as 1). The Energy Dispersive X-ray Spectroscopy (EDX) at the same location reveals, that the surface of the stored cell is enriched with oxygen (green), phosphorus (cyan) and fluorine (blue) (Fig 2c-d). Regarding the cycled cell, the comparison of reference and cycled anode foil reveals spherical and fibrous precipitations [3]. SIMS reveals that (7)Li (Fig. 2f) and (55)Mn (Fig. 2g) are part of the fibrous precipitations (2). Li is enriched in the spherical particles (1) and the electrolyte residual (3). 
The microstructural, analytical examination of the anode surface of aged cells enables the recognition of different ageing fingerprints. SIMS and TOF-SIMS complement the usual analysis methods and offer new insights into ageing mechanisms.

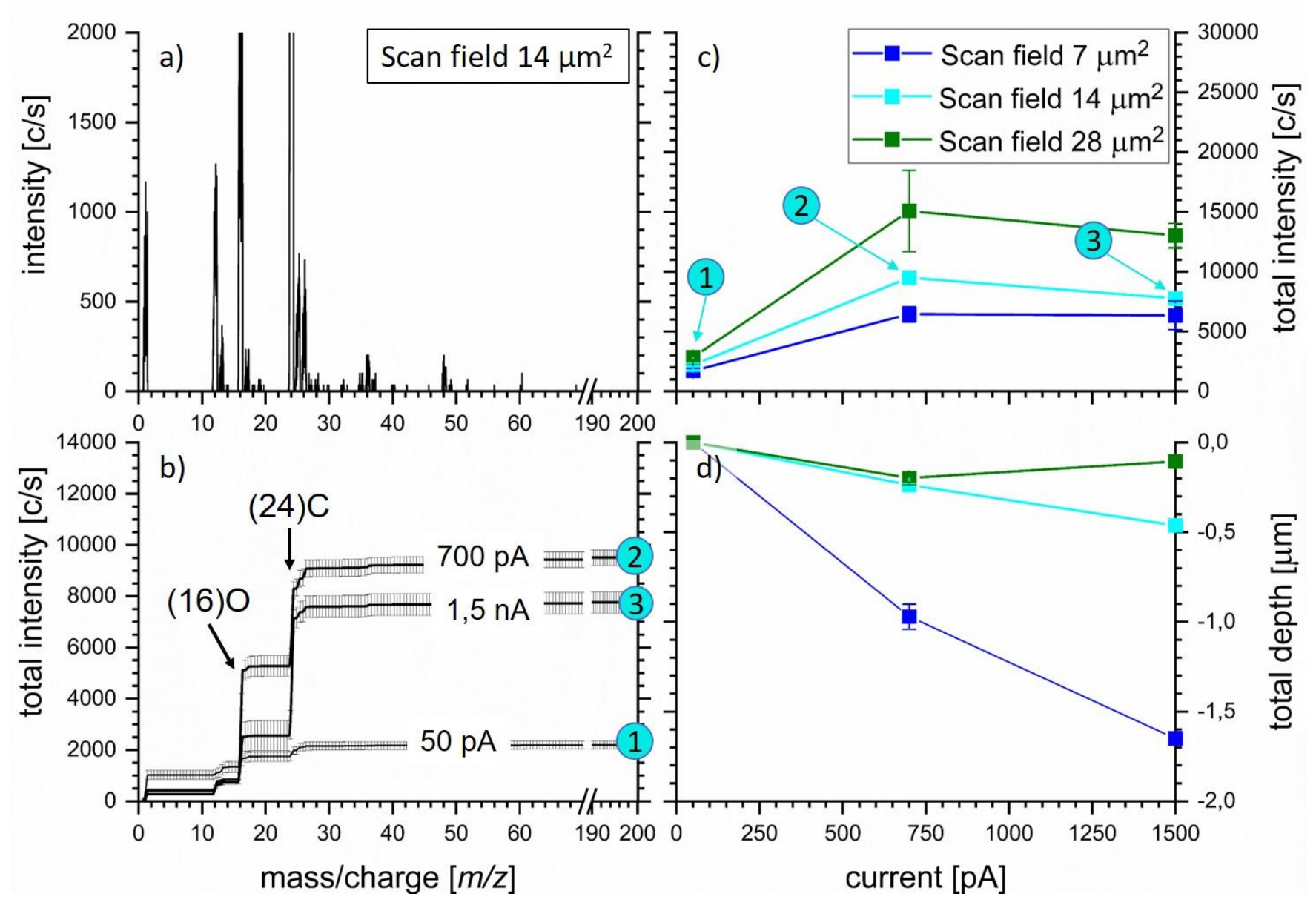

Figure 1. Fig. 1: SIMS of graphite a) spectrum of the negative ions, b) total summed up peak intensities in dependence on the mass/charge for $50 \mathrm{pA}, 700 \mathrm{pA}$ and $1.5 \mathrm{nA}$ beam currents. c) dependence of the total intensity on the scan field size and beam currents. d) total depth of the removed sample in dependence on the scan field and beam currents. the intensity of the elements and the sample removal increases with higher currents; big scanning fields (e.g. $28 \mu \mathrm{m}$ ) show low depth removal also for high intensities 


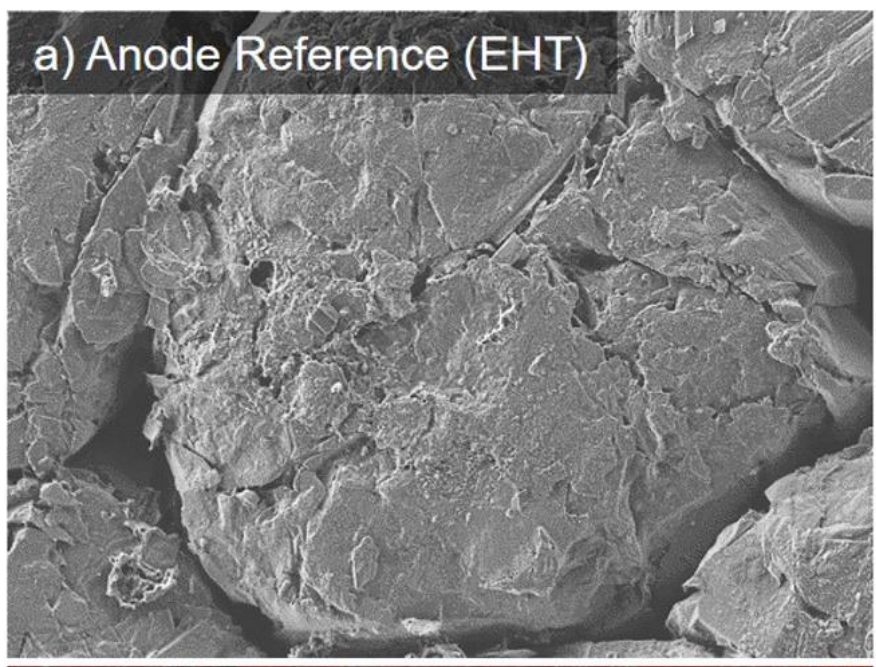

c) Anode Reference (EDX, $5 \mathrm{kV}, 1 \mathrm{nA})$

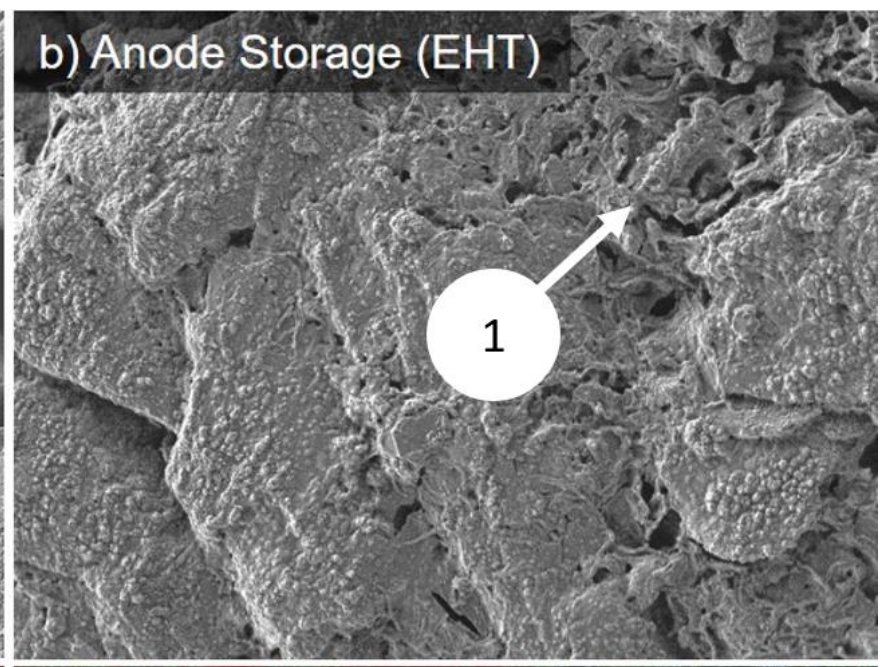

d) Anode Storage (EDX, 5kV,1nA)
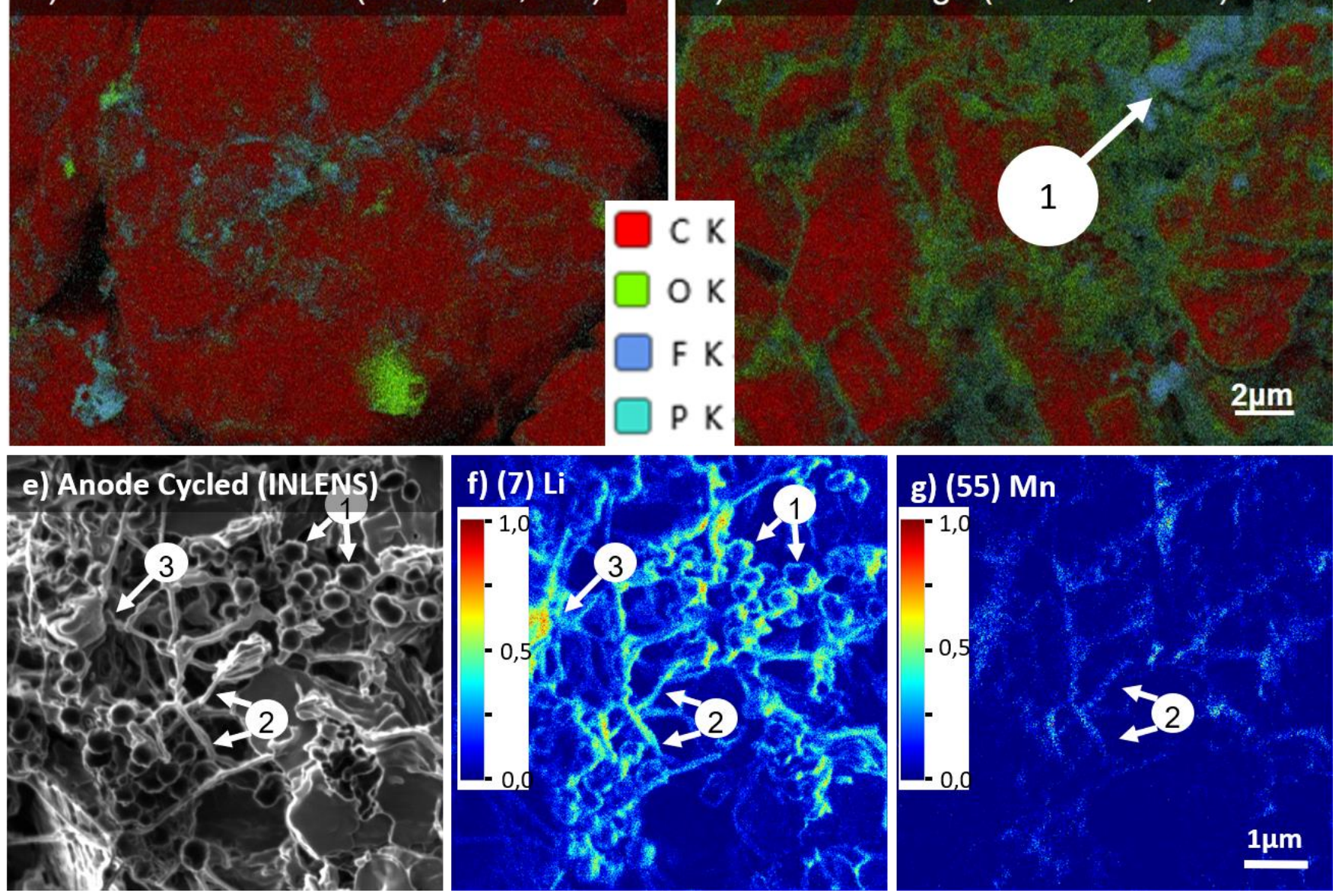

Figure 2. Fig. 2: Surface morphology of the anode (a,b) with corresponding EDX mapping (c,d), deposition products (1) contain oxygen, fluorine and phosphorus; SEM image (e) and element distribution images (positive ions) of (7)Li (f) and (55)Mn (g) (cycled anode foil); spherical (1) and fibrous (2) particles contain (7) Li and (55)Mn; electrolyte residual (3) contains increased (7)Li content; 


\section{References}

[1] Alliata D, Kötz R, Novak P, Siegenthaler H 2000 Electrochemistry Communications 2 436-440.

[2] Vetter J, Novák P, Wagner M R, Veit C, Möller K-C, Besenhard J O, Winter M, Wohlfahrt-Mehrens M, Vogler C, Hammouche A 2005 Journal of Power Sources 147 269-281

[3] Golla-Schindler U, Zeibig D, Prickler L, Behn S, Bernthaler T Schneider G 2018 Micron 113 1019 